\title{
HgCdTe APD- Focal Plane Array development at DEFIR for low flux and photon-counting applications
}

\author{
Johan Rothman', Eric de Borniol, Sylvette Bisotto, Laurent Mollard, Fabrice \\ Guellec
}

CEA/LETI

17 rue des Martyrs - 38054 Grenoble Cedex 9- France

E-mail: johan.rothman@cea.fr

\author{
Frederic Pistone, Solène Courtas, Xavier Lefoule \\ Sofradir \\ 17 rue des Martyrs - 38054 Grenoble Cedex 9- FRANCE
}

\begin{abstract}
We report our latest results obtained on HgCdTe avalanche photo-diodes (APDs) and focal plane arrays (FPAs) manufactured at CEA-LETI in collaboration with Sofradir as a part of our common R\&D effort DEFIR. At DEFIR, e-APDs and dedicated read-out integrated circuits (ROIC) are currently being developed to address both low light passive amplified imaging, to be used, for example, in wavefront correction or fringe tracking in astronomy, and for active laser assisted imaging. The gain, noise and sensitivity performances were measured in planar homojunction APDs with $\mathrm{Cd}$ compositions between $\mathrm{x}_{\mathrm{Cd}}=0.3$ to 0.41 . Exponentially increasing gain, synonym of exclusive electron multiplication, was observed in all the devices up to $M>600$, associated with low noise factors $F=1.2$. Zero-flux measurements showed that the equivalent input dark current, $I_{\text {eq_in }}$, decreases with increasing band-gap, showing that the sensitivity can be optimized in high $\mathrm{x}_{\mathrm{Cd}} \mathrm{e}-\mathrm{APD}$, at the expense of a higher reverse bias which has to be compatible with the ROIC. A record low value of $I_{\text {eq_in }}=2$ aA, corresponding to 12 electrons per second, was obtained in a $\lambda_{\mathrm{c}}=2.9 \mu \mathrm{m}$ e-APD at $M=24$, showing that the e-APD technology is compatible with ultra low flux applications, such as wavefront sensing, which need long integration times. ROICs have been designed for passive and active laser assisted imaging with a format of $320 \times 256$ and a pixel pitch of $30 \mu \mathrm{m}$. A passive imaging ROIC is currently being designed for astronomical applications and aims for a full frame readout speed of $1.5 \mathrm{kHz}$ with an equivalent input noise lower than 2 electrons. Two active imaging ROICs have already been validated with e-APD arrays. A dual mode passive and active 2D (range gated) FPAs have been made using eAPDs with cut-off wavelengths ranging from $2.9 \mu \mathrm{m}$ to $5.3 \mu \mathrm{m}$ at $T=80 \mathrm{~K}$. On the best devices, the operability in gain and noise exceeds $99.6 \%$ and relative gain dispersion lower than $10 \%$, independently of the wavelength at gains $M=10-100$. Finally, the exclusive electron multiplication in the e-APDs is shown to enable linear mode photon counting at high gain and low dark count rate, associated with a low timing jitter $\Delta t<100$ ps.
\end{abstract}

Quantum of Quasars workshop

Grenoble, France

December 2-4, 2009

\footnotetext{
$1 \quad$ Speaker
} 


\section{Introduction}

In recent years, $\mathrm{HgCdTe}$ APDs have been demonstrated to be one of the most promising paths to Focal Plane Arrays for low flux and high speed applications such as active and hyper spectral imaging. Several groups [1-18] have reported exponentially increasing multiplication gains of 100-1000 for low values of reverse diode bias (5-10 V on the n-type contact), associated with a nearly conserved signal to noise ratio SNR with an excess noise factor $F=\mathrm{SNR}_{\text {in }} / \mathrm{SNR}_{\text {out }}$ close to unity. This behaviour contrasts with the one observed in III-V material or Si APDs, which requires high reverse bias and have typical noise factors of $F_{\text {III-V }} \sim 4-5$ and $F_{\mathrm{Si}}$ 2-3, respectively [19]. These exceptional characteristics of $\mathrm{HgCdTe}$ APDs are due to a nearly exclusive impact ionization of the electrons, explaining why theses devices have been termed electrons initiated avalanche photodiodes, e-APDs.

Recently, we have shown that the exclusive multiplication of electrons also has a strong implication on the response time and bandwidth of the APDs [15-18]. The bandwidth in III-V material or Si APDs, in which both electrons and holes contribute to the multiplications, is known to be inversely proportional to the gain. This is due to high multiplication events with slow evacuation of carriers from the depletion layer. As a consequence, the gain-bandwidth product, $G B W$, saturates at high gains and a maximum value of $320 \mathrm{GHz}$ has been achieved in III-V APDs [19]. In contrast, a nearly constant and resistance-capacitance $(R C)$ limited bandwidth and a record high $G B W=2.1 \mathrm{THz}$ have been demonstrated in $\mathrm{HgCdTe}$ mid-wave (MW) infrared e-APDs [18], in consistency with theoretical predictions of constant $B W$ for unipolar multiplication in APDs [20]. In addition, the gain-bandwidth limit given by the carrier transit time through the depletion layer was shown to be $G B W \sim 20 \mathrm{THz}$ in a $4 \mu \mathrm{m}$ wide depletion region. This property of the HgCdTe e-APDs shows that a high bandwidth, $B W>10 \mathrm{GHz}$, can be obtained in optimised devices even at high gains $M>100$. In addition, the exclusive quasi-deterministic electron multiplication implies that the response time is reproducible and that the multiplication process does not produce excessive timing jitter. Hence, for time resolved photon counting applications with $\mathrm{HgCdTe}$ e-APDs, the timing jitter is mainly limited by collection of minority carriers into the multiplication region. This contribution will be further discussed in section 4 of the present communication.

The high gain at low bias, the low noise factor, and high bandwidth product makes the $\mathrm{HgCdTe}$ APDs particularly well suited for integration in the next generation FPAs. They will enable new applications that require high sensitivity and/or fast detection, such as active and passive amplified imaging. The latter is of particular interest for low flux applications in the MWIR range, observing in a narrow field of view or spectral range, and in the short wave infrared (SWIR) range, for high frame-rate applications requiring sub-photon read-out-noise, such as wave-front sensing and fringe tracking. The amplification of the photocurrent can improve the linearity of some ROIC designs and a dynamic gain could be used to increase the dynamic range. The high and homogeneous gain and high speed of the e-APDs do also make them an ideal candidate for photon counting applications, for example to suppress read-out 
noise and/or to perform time-stamped measurement for single molecule fluorescence measurements and quantum optics.

Since the first demonstrations of the exponential gain and ultra low excess noise factor by Beck et al. at DRS [1-4], the high potential of HgCdTe e-APDs have been confirmed by several groups [6-18], and first results on dedicated e-APD FPAs were published recently. Baker et al. at Selex were the first to demonstrate laser gated imaging in a 320x256 $24 \mu \mathrm{m}$ pitch APD FPA [10]. They reported avalanche gains up to $M=100$, low excess noise and an input noise equivalent photon noise $N E P h=15 \mathrm{rms}$, for a short integration times $t_{i n t}=1 \mu \mathrm{s}$ for $\lambda_{c}=4.2 \mu \mathrm{m}$ APDs. This corresponds to an equivalent input dark current of about $I_{\text {eq_in }}=40 \mathrm{pA}$, which was partly limited by a residual photon-flux. Later [11, 12], they described the interest of a dual mode active passive imaging and reported first results on 3D active imager with a range resolution of $1 \mathrm{~m}$. A $99 \%$ operability (number of functional APDs) was also reported, but the criteria was not clearly stated. In 2007, Beck et al. at DRS reported their first realisation of a

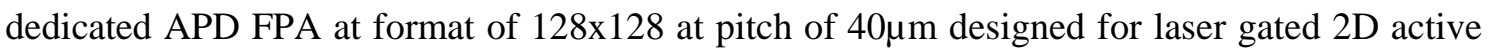
imaging with integration times between $50 \mathrm{~ns}$ and $500 \mu$ s [13]. The ROIC was designed for dual mode passive and active imaging using two capacitors. They reported high maximum gains $M=300-950$ associated with $99 \%$ operability in gain, defined as the number of functional pixels in a range of $+/-50 \%$ of the average gain $\langle M\rangle$, and low $N E P h<5$ at $t_{i n}=1 \mu$ s. Both of the above demonstrations were realised with e-APDs manufactured using liquid phase epitaxy (LPE) grown absorption layers with a Cd composition corresponding to a cut-off wavelength of $\lambda_{\mathrm{c}}=4.2-5.0 \mu \mathrm{m}$ at $T=80 \mathrm{~K}$.

In this communication, we report recent developments of e-APDs and e-APD-FPAs at DEFIR, the research collaboration of CEA-LETI and Sofradir. The avalanche gain performance of planar e-APD with cut-off wavelengths ranging from $\lambda_{\mathrm{c}}=2.9-5.3 \mu \mathrm{m}$ have been studied to establish the potential to optimize the e-APDs in the gain-sensitivity-spectral bandwidth-ROIC compatibility parameter space. In particular, the increase in band-gap yields a lower thermally generated dark current at higher temperature, but requires a higher reverse bias to obtain a given multiplication gain, which can be difficult to obtain with traditional CMOS technologies. The avalanche gain and noise performance, measured on test arrays, is reported in the section 2.2. In the following section we discuss the e-APD FPA developments at DEFIR aiming for active and

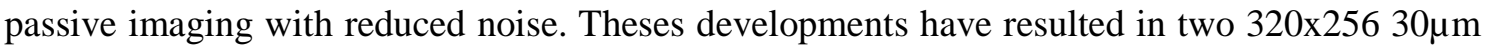
pitch FPAs designed for dual-mode passive and 2D active imaging and single shot 3D/2D active imaging. The gain, noise and sensitivity performance of e-APDs arrays with cut-off wavelength $\lambda_{\mathrm{c}}=2.9-5.4 \mu \mathrm{m}$ have been characterised at $T=80 \mathrm{~K}$ using the dual-mode ROIC. These results are reported in sections 3.1. The single shot 3D active imaging FPA will not be discussed here, although it is a first experience in designing a faint event driven FPA, which is nessecary for time resolved photon counting. The potentials of using the CEA-LETI HgCdTe e-APDs for photon counting applications are however discussed in section 4. 


\section{Homojunction e-APD Optimization}

\subsection{Device technology}

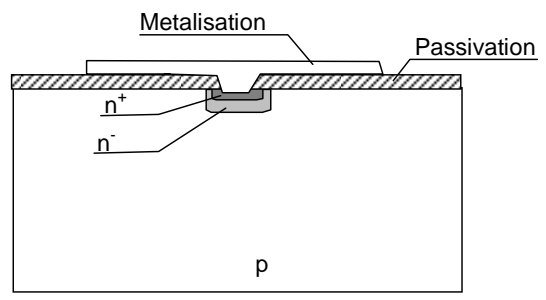

Figure 1. Schematic illustration of a planar homo-junction CEA/LETI HgCdTe e-APD

The devices were processed on wafers grown by molecular beam epitaxy, MBE, or Liquid Phase Epitaxy, LPE. The absorption layers in the present study had $\mathrm{Cd}$ compositions ranging from $\mathrm{x}_{\mathrm{Cd}}=0.3$ to $\mathrm{x}_{\mathrm{Cd}}=0.41$. An increase in $\mathrm{x}_{\mathrm{Cd}}$ gives and increased band-gap and a corresponding reduction of the cut-off wavelength $\left(\lambda_{c}\right)$. At wavelengths shorter than $\lambda_{c}$, the light can be effectively absorbed by the semi-conductor. In HgCdTe diodes, a high a close to constant quantum efficiency have demonstrated from visible wavelengths up to $\lambda_{\mathrm{c}}$. When $\mathrm{x}_{\mathrm{Cd}}$ varies from 0.3 to $0.4, \lambda_{c}$ reduces from 5.3 to $2.9 \mu \mathrm{m}$ at $T=80 \mathrm{~K}$.

The HgCdTe MBE growth was carried out in a RIBER 2300 system using (211)B $\mathrm{Cd}_{0.96} \mathrm{Zn}_{0.04} \mathrm{Te}$ substrates. Individual effusion cells were used for the evaporation of cadmium, tellurium and mercury species. The growth temperature is about $180^{\circ} \mathrm{C}$. The mercury flux is kept constant during the epitaxy of the whole structure. Careful adjustment and monitoring of both substrate temperature and mercury flux is crucial to prevent any degradation of the material quality.

The LPE grown devices were elaborated using the slider technique. Growth is performed in the tellurium-rich corner of the phase diagram at a temperature of $470{ }^{\circ} \mathrm{C}$ and with a (111)B crystalline orientation. The LPE samples had a cut off wavelengths $\lambda_{c}=5.2-5.3 \mu \mathrm{m}$ at $T=80 \mathrm{~K}$.

The figure 1 shows a schematic illustration of a LETI HgCdTe e-APD. A pin junction is formed by transforming a narrow region close to the surface in a vacancy doped $\mathrm{p}$ type layer $\left(N_{a}=3 \times 10^{16} \mathrm{~cm}^{-3}\right)$, into an $\mathrm{n}^{+}$region with a doping level of $N_{d}=1 \times 10^{18} \mathrm{~cm}^{-3}$. The formation of the $\mathrm{n}^{+}$will also generate an $\mathrm{n}^{-}$layer by the suppression of $\mathrm{Hg}$ vacancies. The doping of the $\mathrm{n}^{-}$region is due to the residual doping of the epitaxy and is typically $N_{d}=3 \times 10^{14} \mathrm{~cm}^{-3}$. The extension of the $\mathrm{n}$ - layer is correlated to the depth of the $\mathrm{n}^{+}$layer. The width of the $\mathrm{n}^{-}$layer in the devices has not yet been determined but is expected to in the order of $1-3 \mu \mathrm{m}$. 


\subsection{Gain Measurements in $\mathrm{x}_{\mathrm{Cd}}=0.3-0.41 \mathrm{HgCdTe}$ e-APDs}

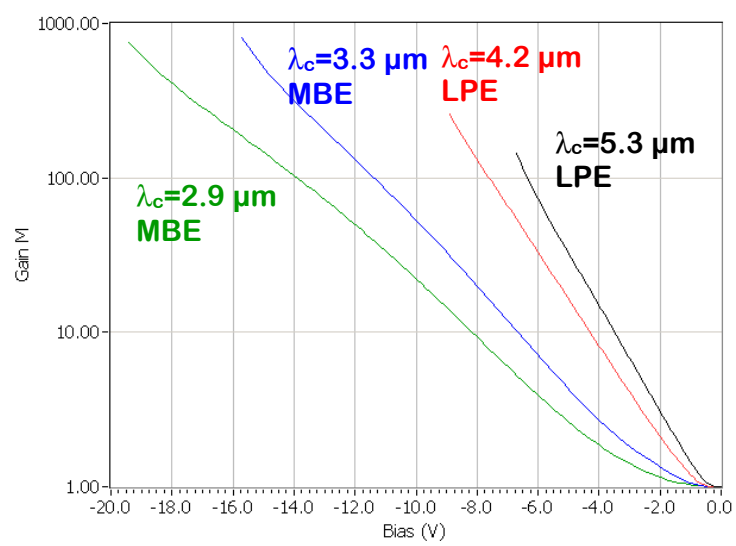

Figure 2. HgCdTe e-APD gain curves measured at $T=80 \mathrm{~K}$ for $\lambda_{c}=2.9 \mu \mathrm{m}$ to $5.3 \mu \mathrm{m}$.

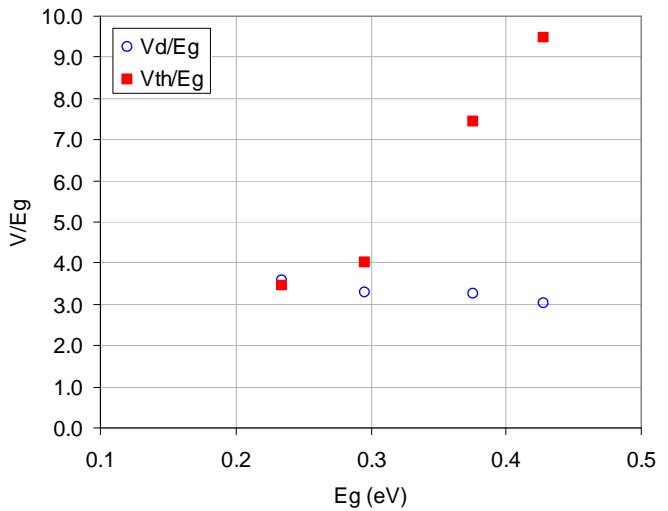

Figure 3. Energy gap dependence of the phenomenological gain parameters $V_{d}$ et $V_{\text {th }}$ estimated at $T=80$ $K$ at gains between $M=10$ and 100 .

Typical gain curves measured at $T=80 \mathrm{~K}$ for e-APDs with $x_{\mathrm{Cd}}$ ranging from $x_{\mathrm{Cd}}=0.3$ to $x_{\mathrm{Cd}}=0.4$ and similar junction geometry are reported in figure 2 . As the Cadmium composition increases, the gain at a given reverse bias decreases due to the increase in band-gap. An exponentially increasing gain is observed for all compositions up to gains higher than $M=100$, showing that the exclusive electron multiplication is stable at high gains even in shortwavelength devices. For the diode with the shortest cut-off wavelength, $\lambda_{\mathrm{c}}=2.9 \mu \mathrm{m}$, gains up to $M=600$ were obtained at a reverse bias of $V_{\text {bias }}=20 \mathrm{~V}$. To the best of our knowledge, this is the highest gain value that has been measured in SW HgCdTe e-APDs.

The variation of the gain as a function of band-gap was quantified using Becks phenomenological formula [2]:

$$
M=2^{\frac{V-V_{t h}}{V_{d}}}+1
$$

where $V_{\text {th }}$ is a threshold voltage which corresponds to the potential across the junction which is needed to generate a first multiplication event. $V_{\mathrm{d}}$ is the additional voltage needed to initiate another multiplication event and hence to double the value of the gain. The voltage dependence of the gain has been quantified by fitting the Beck formula at intermediate gain values $M=30$ to 50 . The estimated values of $V_{\text {th }}$ and $V_{\mathrm{d}}$, normalised by the band gap are reported 
as a function of the band gap in figure 3. It can be seen that the $V_{\mathrm{d}}$ is fairly independent of the Cd composition, but $V_{\text {th }}$ increases strongly. This behaviour can be correlated with the increase in effective mass at higher $\mathrm{xCd}$, which modifies the scattering mechanisms of the electrons. It should however be emphasized that the increase in $V_{\text {th }}$ could also be caused by a change in junction geometry; an increase of the depletion layer width has been found to be correlated with an increase in $V_{\text {th }}$ at constant $x_{\mathrm{Cd}}[15]$.

The exclusive electron multiplication is corroborated by the observation of a low excess noise factors down to the shortest examined wavelengths. This is illustrated in figure 4, in which the noise current spectral density is plotted for reverse biases of $0.1 \mathrm{~V}, 5 \mathrm{~V}, 10 \mathrm{~V} 15 \mathrm{~V}$ and $20 \mathrm{~V}$, corresponding to gains $M=1,2,10,60$ and 600 , respectively. The noise spectra at each bias is compared with the expected shot-noise limited signal in the case $F=1$. It can be seen that an excess noise factor close to unity is obtained at all biases. The typical average value between $f=10-30 \mathrm{~Hz}$ is $F=1.2$, which is similar to the values obtained longer wavelength devices $[1-3,15$, $21,22]$.

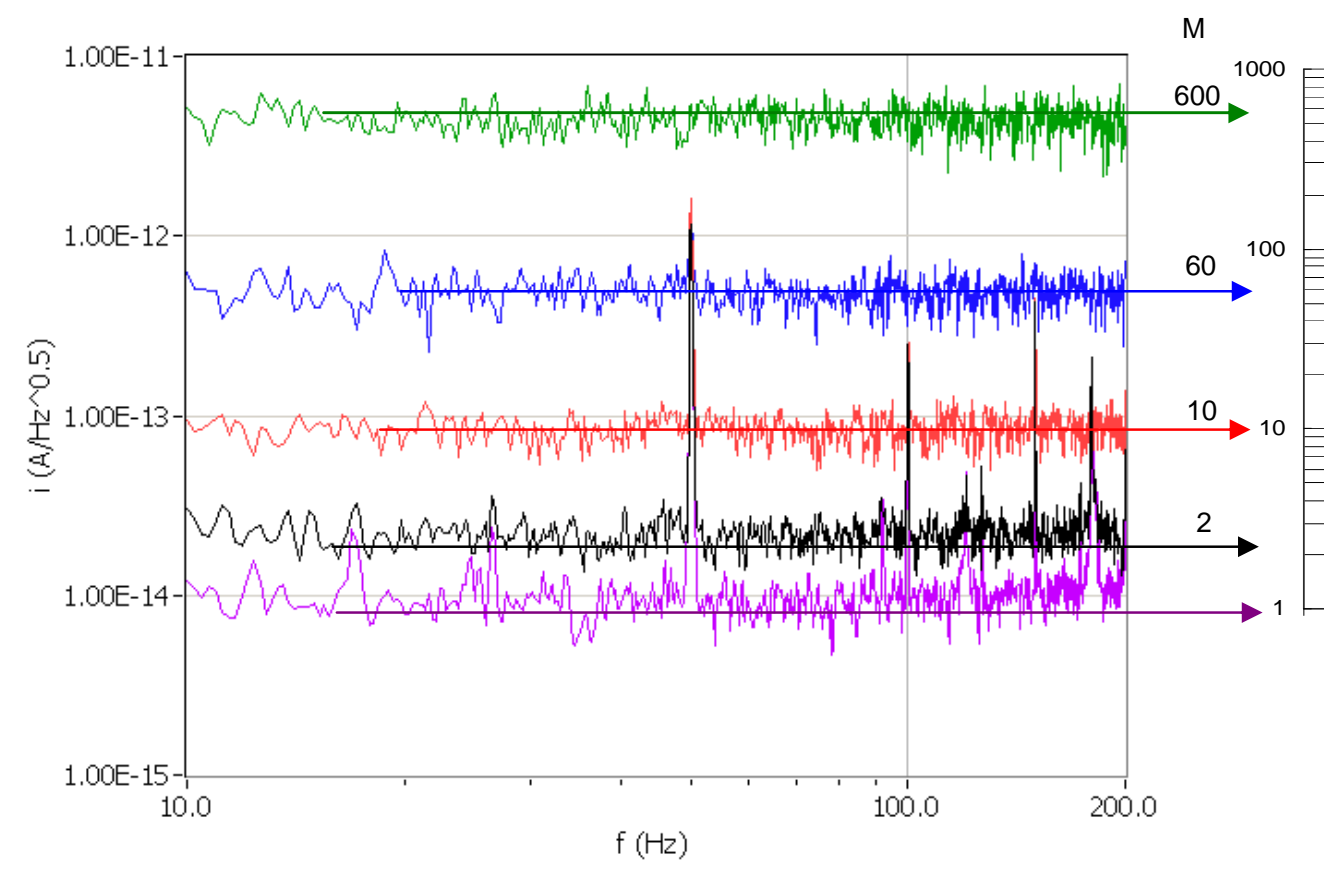

Figure 4. Evolution of the current noise spectral density as a function of the gain in a $\lambda_{c}=2.9 \mu \mathrm{m} \mathrm{HgCdTe}$ $e-A P D$ at $T=80 \mathrm{~K}$. The arrows indicate the expected shot-noise limited noise at the different values of the gain.

\section{HgCdTe e-APD FPA developments at Defir}

To benefit from the advantages enabled by $\mathrm{HgCdTe}$ e-APDs in FPAs, specifically designed ROICs capable of applying a high reverse bias need to be developed. At DEFIR, we are currently developing three different ROIC architectures aiming for dual-mode passive-active $2 \mathrm{D}$ imaging, single-shot simultaneous 3D/2D imaging and passive amplified ultra-fast imaging. The dual-mode passive and active imaging FPA was first demonstrated using a MWIR e-APD array $[23,24]$. In the present communication, theses results have been corroborated with new measurements on e-APD FPAs with shorter cut-off wavelengths and with zero-flux 
measurements that allow estimating the sensitivity limit of the e-APDs. The ultra-fast passive imaging FPA is developed for astronomical applications such as wave-front sensing and fringe tracking at a frame rate higher than $1.5 \mathrm{kHz}$ and a total noise lower than 2 electrons per frame. The format of this FPA is 320x256 (TV/2) with a pixel pitch of $30 \mu \mathrm{m}$. To achieve this performance, a high gain and low dark current is required. The potential of the e-APD arrays for theses applications is discussed in section 3.1, in view of dark current measurements performed using the dual-mode e-APD FPAs with varying Cd composition of the e-APD arrays. The single shot 2D/3D array is an extension of a first event driven small test array $(4 \times 10)$ [25], which have been extended into a larger TV/2 format. This FPA is currently under test.

In parallel with theses large format developments, we are currently investigating Si CMOS electronics to take benefits of the potentials of e-APDs for photon-counting applications, with or without timing resolution. Theses potentials are discussed in section 4 .

\subsection{HgCdTe e-APD-FPA for dual-mode passive/active imaging}

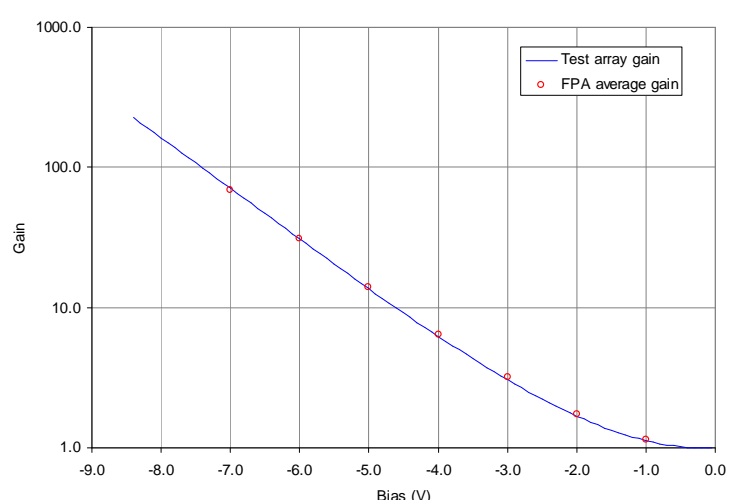

Figure 5. Average gain in a $2 D A P D-F P A$ compared the gain estimated from $I(V)$ measurement on an APD on a test array. Both measurements were performed at $T=80 \mathrm{~K}$.

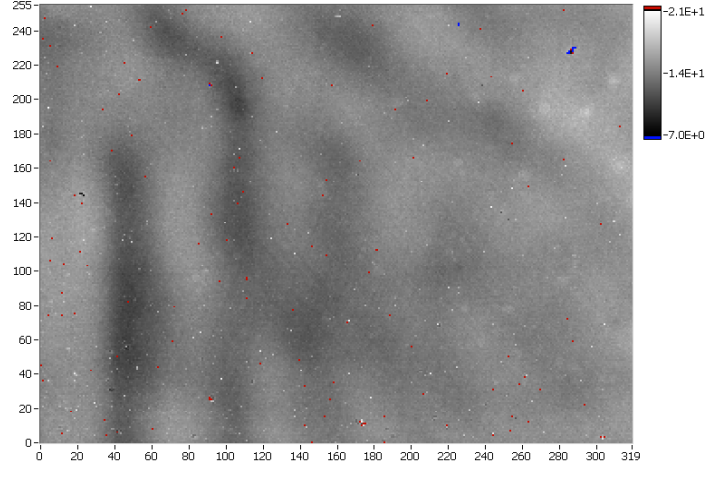

Figure 6. Gain map measured at 5 V reverse bias in the dual mode APD-FPA. The red and blue levels corresponds to defect pixel out of the range.

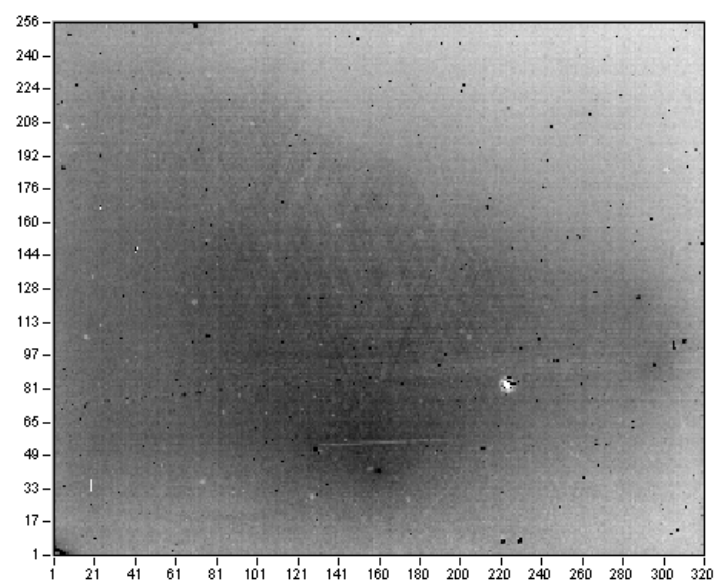

Figure 7. Continuous level (out-put voltage) map of a $\lambda_{c}=3.3 \mu \mathrm{m}$ at a reverse bias of $V_{b}=7 \mathrm{~V}(M=10)$ at $T=80 \mathrm{~K}$. The operability at $\langle C L\rangle+/-30 \%$ is $99.8 \%$

The dual-mode passive active imaging APD-FPAs have been used to characterize the gain, noise and sensitivity performance of $\mathrm{HgCdTe}$ e-APD FPAs with $\mathrm{Cd}$ composition varying from 
$\mathrm{x}_{\mathrm{Cd}}=0.41-0.3$. The FPAs have a format of $320 \times 256$ and a pixel pitch of $30 \mu \mathrm{m}$. Each pixel includes two capacitors to integrate the signal, with integration times down to $t_{\mathrm{int}}=100 \mathrm{~ns}$. A first capacitor $C_{0}=30 \mathrm{fF}$ is used for active mode $2 \mathrm{D}$ imaging, with a total measured read-out noise that has been $\Delta N_{\mathrm{C} 0}=90$ electrons rms. Using this capacitor, the equivalent read-out-noise will be equal to 1 for gain $M>90$. A second capacitor $C_{1}=120 \mathrm{fF}$, can be used for passive imaging with or without gain, with a measured read-out noise $\Delta N_{\mathrm{Cl}}=150$ electrons rms. The characteristics of the dual-mode ROIC are further described in reference [24].

A first MWIR e-APD-FPA was characterized at $T=80 \mathrm{~K}$. Measurements were preformed with a photon flux at $\mathrm{f} / 2$, using black-body temperatures ranging from $20^{\circ} \mathrm{C}$ and $30^{\circ} \mathrm{C}$, and at $0^{\circ}$ FOV. At low bias $(50 \mathrm{mV})$, the operability in photon induced current $I(+/-30 \%)$ and noise $(+/-$ $50 \%$ ) was $99.96 \%$, associated with an external quantum efficiency of $\eta=70 \%$. This result confirms that the LETI e-APD technology is well suited to produce state of the art passive imaging and enables high performance dual-mode active passive imaging. In addition, the APDFPA was found to be fully functional up to the maximum bias enabled in the set-up (7V). Figure 5 compares the average gain in the APD-FPA with a gain curve for a large junction area APD on a test-array from the same wafer. The good agreement between the data demonstrates the capacity of the 2D-APD-FPA to integrate the amplified photo-current and, yet again, the low gain dispersion in $\mathrm{HgCdTe}$ e-APDs.

The high homogeneity of the gain is illustrated in figures 6, which report a gain map measured at $5 \mathrm{~V}$ reverse bias. The relative gain dispersion at this gain was $\sigma_{\mathrm{M}} / M=7.7 \%$, which is lower than the dispersion observed on the non amplified current, $\sigma_{\mathrm{I}} I>9 \%$ at $50 \mathrm{mV}$ reverse bias. It can be seen that the main contribution to the dispersion is long range fluctuations induced by the LPE growth, so called ripples. Hence, the dispersion should be reduced in absorption layers grown by MBE or LPE with reduced ripple. A $99.8 \%$ operability in gain at $\langle M\rangle=+/-50 \%$ was observed up to the maximum reverse bias of $7 \mathrm{~V}$, corresponding to $M=70$. This high operability demonstrates the capability of high performance amplified passive and active imaging using $\mathrm{HgCdTe}$ APD-FPAs. This conclusion is corroborated by the measurements of the excess noise factor, which was found to be $\langle F\rangle=1.4$, associated with an operability of $99.7 \%$ up to the maximum gain $M=70$. The operability in $F$ is higher than the one observed on test-arrays. This indicates that the defects in excess noise are highly dependent on the local environment of the APDs and that the operation of the APDs is favored by a high proximity of the read-out electronics, i.e. when hybridized to an FPA.

Similar results have been obtained in SWIR e-APD FPAs, realized using MBE grown epilayers. The figure 7 reports the un-corrected continuous level map measured at a reverse bias of $V_{\mathrm{b}}=7 \mathrm{~V}$ in a $\lambda_{\mathrm{c}}=3.3 \mu \mathrm{m}$ e-APD FPA at $T=80 \mathrm{~K}$. At this bias the gain is close to $M=10$ and the operability at $+/-30 \%$ of the average value is $99.8 \%$. The operability at $+/-50 \%$ of the average noise was equally high at $99.6 \%$, with an average noise corresponding to an excess noise factor $\langle F\rangle=1.2$. Theses results shows that our e-APD technology is compatible with the manufacture of high operability e-APD arrays using MBE or LPE grown $\mathrm{HgCdTe}$, with cut-off wavelengths ranging from the SWIR to the MWIR spectral bands. Hence, the Cd composition can be tuned as a function of the required wavelength, gain and sensitivity. 
The sensitivity of the e-APDs is best determined from zero-flux measurements in which the detected charges are not generated by photons. In MWIR e-APDs, it is possible to estimate $I_{\text {eq_in }}$ from measurements with residual photon-flux by subtracting short circuit current $I_{\mathrm{cc}}$ $[15,23]$. In the present SWIR e-APDs, the residual photon contribution is lower than $I_{\mathrm{cc}}<10 \mathrm{fA}$ and can not be correctly estimated due to shunt resistances in the experimental set-up. At present, only a $\lambda_{\mathrm{c}}=2.9 \mu \mathrm{m}$ e-APDs test array have been characterized in a zero-flux open-cycle He flow cryostat. The median dark current was however so low that $I_{\text {eq_in }}$ could only be measured at a reverse bias of $V_{\mathrm{b}} \geq 19 \mathrm{~V}$ ( $M \geq 380$ ). At $M=380$ we measured $I_{\text {eq_in }}=1.5 \mathrm{fA}$. This value is compared to earlier reported values [23], measured at $M=100$ and $T=80 \mathrm{~K}$ in MW eAPDs in figure 8 . It can be seen that $I_{\text {eq_in }}$ in the SW e-APD is slightly higher than the trend-line extrapolated from the MW e-APD values. The higher $I_{\text {eq_in }}$ could be related to the higher gain, which tends to increase the dark current. Zero-flux measurements have also been realised at lower gain using the high sensitivity of the 2D e-APDs FPA and integration times up to $50 \mathrm{~s}$. The good diode median values of $I_{\text {eq_in }}$ in MWIR and SWIR e-APD FPAs are compared to the high gain test array results in figure 6 . In the MW e-APD, $I_{\text {eq_in }}$, reduces by more than one order of magnitude when the gain is reduced from $M=100$ to $M=10$, yielding $I_{\text {eq_in }}(M=10)=10 \mathrm{fA}$. In a $\lambda_{\mathrm{c}}=3.3 \mu \mathrm{m}$ e-APD FPA, the median equivalent dark current at $M=6$ was higher than the MW eAPD trend line; $I_{\text {eq_in }}=96 \mathrm{aA}$. However, at $\lambda_{\mathrm{c}}=2.9 \mu \mathrm{m}$ at $M=24$, the equivalent dark current was only $I_{\text {eq_in }}=2 \mathrm{aA}$. This value corresponds to 12 electrons per second and is below the $M=100$ trend-line and is the lowest dark current reported so far in any type of APDs. This result shows that $\mathrm{HgCdTe}$ e-APDs can be used for ultra-low flux applications, such as wave-front correction and fringe-tracking in astronomy.

Finally, despite the dispersion in sensitivity observed in figure 8, the $M=100$ trend line seems to give a rough estimation of the dark current in the e-APDs down to cut-off wavelengths $\lambda_{\mathrm{c}}<3.0 \mu \mathrm{m}$. The trend line indicates that equivalent input currents of the order of 1 electrons $/ \mathrm{s}$ should be achievable in e-APDs with cut-off wavelength shorter than $\lambda_{\mathrm{c}}<2.5 \mu \mathrm{m}$ and at gains $M>10$.

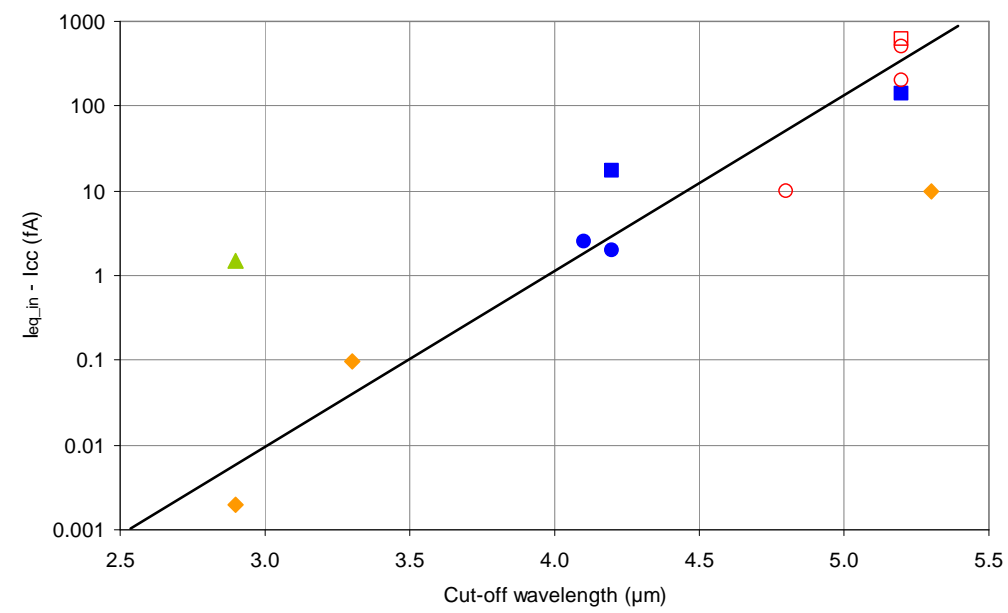

Figure 8. Equivalent input current at $T=80 \mathrm{~K}$ in e-APDs test arrays at $M=100$ (circles and squares) and $S W$ and $M=380$ (triangle,) and ROIC measurements with M=6-24 (diamonds). The open symbols represent measurements made with residual photon flux $I_{c c}$, which have been subtracted from the measured $I_{\text {eq_in }}$. The full symbols represent measurements at zero flux, i.e. with $I_{c c}=0$. 


\section{HgCdTe e-APD-FPA for photon counting applications}

The exclusive electron multiplication in $\mathrm{HgCdTe}$ e-APDs makes them ideal candidates for photon-counting applications. The close to deterministic multiplication implies that the response time is expected to be independent on the multiplication gain [20]. We have confirmed this in two studies $[17,18]$, in which we demonstrated record high bandwidth product for amplified photo-detectors, $G B W=2.1 \mathrm{THz}$. In addition, the estimation of the saturation of the carrier velocities in the un-doped depletion layer showed that values close to $G B W=20 \mathrm{THz}$ can be reached in optimised structures. In another publication [26], we measured the variation of the drift velocity in p-type doped $\mathrm{HgCdTe}$ material, hence in the region where the carriers are absorbed and need to either diffuse or drift to the multiplication region before the event can be detected. As the multiplication is quasi-deterministic, this collection of the photo generated minority electron will give a dominant contribution to the timing jitter in the detectors. If the photons are absorbed in a layer with a built-in electrical field that direct the photo-electrons to the multiplication layer, the timing jitter of the detector will be directly related to the thickness of the absorption layer and the drift velocity. This study allowed us to estimate the timing jitter in a $4 \mu \mathrm{m}$ thick absorbing region, $\Delta t_{\mathrm{jitter}}=50-100 \mathrm{ps}$, depending on the composition of the material and the built in electrical field. The timing jitter is an important parameter for the quantum time correlation measurements, as it is inversely proportional to the spectral resolution, $\Delta E(e V)=1.24 \Delta \lambda \lambda^{2}$, and, as a consequence, to the flux within $\Delta \lambda$. A timing resolution of around $\Delta t=100 \mathrm{ps}$ is commonly requested to obtain a sufficient photon flux and consequently, to establish photon statistics in a reasonable time. Hence, the timing resolution of $\mathrm{HgCdTe}$ eAPDs, in optimized structures, respond to the demands of most time resolved photon counting applications.

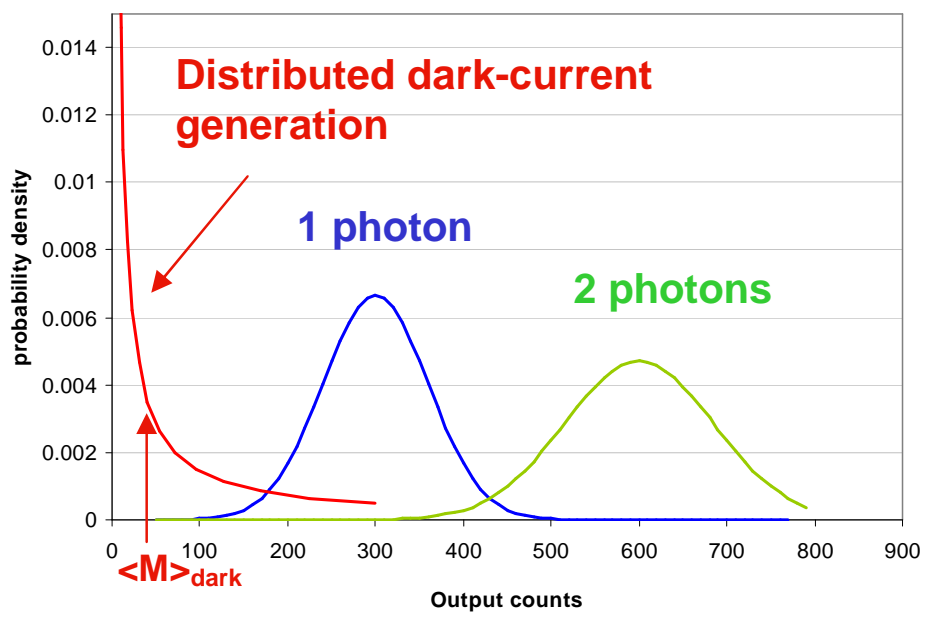

Figure 9. Multiplication gain distributions for 1 and 2 photons events and for a uniformly distributed dark current generation in the multiplication layer.

Another aspect of the exclusive and low noise multiplication in $\mathrm{HgCdTe}$ e-APD is illustrated in figure 9. This figure compares the multiplication gain distribution for one and two simultaneous initial electrons (photons). The gain distributions have been calculated supposing 
a Gaussian distribution of the gain with a standard deviation given by the excess noise factor of the multiplication process:

$$
F=1+\frac{\sigma_{M}^{2}}{\langle M\rangle^{2}}
$$

where $\langle M\rangle$ is the average gain. The 1 and 2 photon distributions in figure 9 have been plotted for an $F=1.04$. For this value of $\mathrm{F}$, the two distributions are well separated and proportional photon counting can be achieved by thresholding at an intermediate multiplication value, for example $M_{2 \_ \text {photons }}>430$. It should be stressed that this value of $F$ is lower than the one measured on the planar APD, $F_{\text {mes }}=1.1-1.4$. However, the measured excess noise factors include un-amplified photon generation in the multiplication layer, which leads to an overestimation of the excess noise factor. The actual value of the excess noise factor in HgCdTe e-APD with photon absorption out-side the junction is still to be determined. Still, one can see that even for an excess noise factor $F=1.04$, the distribution is wide. Hence, for the e-APD allow proportional photon counting, i.e. to be able to distinguish between 1 or 2 photon events, the excess noise factor needs to be very low $F<1.2$.

The gain distribution for 1 and 2 photons events is also compared with the distribution of multiplication counts for one dark current event, which probability distribution of the position of generation is constant in the multiplication layer. Supposing a gain which is increasing exponentially as a function of the distance in the layer, one can show that the average dark current gain can be expressed as a function of the total gain through the multiplication layer, $M_{\text {diff: }}$

$$
\langle M\rangle_{\text {dark_cont_gen }_{-}}=\frac{M_{\text {diff }}}{\ln \left(M_{\text {diff }}\right)}\left(1-\frac{1}{M_{\text {diff }}}\right)
$$

This example shows that some the dark current events, the dark counts, can be excluded using a threshold, for example at a level $M_{1-\text { photon }}=150$. The efficiency of the dark current thresholding will depend on the value of the excess noise factor and the actual distribution of the generation of dark current events. Information on the latter can be obtained from dark current noise measurements made with zero flux, by comparing the estimated equivalent input dark current, $I_{\text {eq_in, }}$, with the actual measured dark current, supposing that both are shot noise limited and the $F \sim 1$. Knowing the total diffusion current gain (the photon gain, $M_{\text {diff }}$ ), it is possible to estimate the average dark event gain:

$$
\left\langle M_{\text {dark }}\right\rangle=\frac{M_{\text {diff }}^{2} I_{e q_{-} i n}}{I_{\text {dark }}}
$$

In the figure 10 , we compare $I_{\text {eq_in }}$ with $I(V)$ curves measured on a $\lambda_{\mathrm{c}}=5.4 \mu \mathrm{m}$ e-APD at $T=80 \mathrm{~K}$ and different conditions of illumination. In table $\mathrm{x}$ is reported the values $\left\langle M_{\mathrm{dark}}\right\rangle$ and

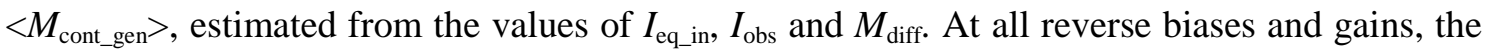
average dark current gain is lower than the diffusion gain, indicating that a part of the dark current should be efficiently thresholded out. At low gain, the average dark current is comparable with the continuously distributed dark current generation. At higher reverse bias, at which the steep increase in dark current has been attributed to tunneling currents [15], the 
average dark gain tends to unity. This result gives an indication that the tunneling current is generated close to the end of the multiplication layer and that it is only weakly amplified. If this result is confirmed, the tunneling current in our e-APDs can be threshold out even for low values of $M_{1_{-} \text {photon, }}$, which will enable photon counting at high gain and low dark count rates.

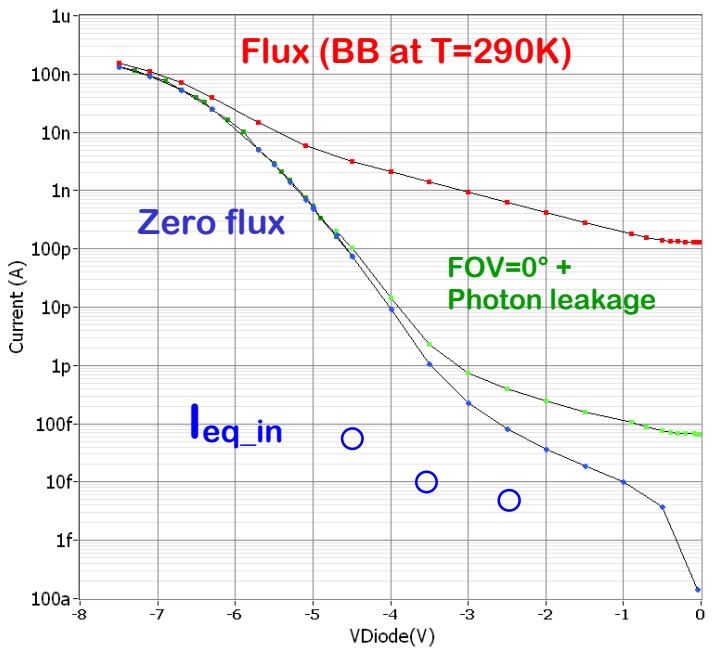

Figure 10. I(V) and $I_{\text {eq_in }}$ in a $\mathrm{HgCdTe}$ e-APD with $\lambda_{c}=5.4 \mu \mathrm{m}$ at $\mathrm{T}=80 \mathrm{~K}$.

\begin{tabular}{|c|c|c|c|c|c|}
\hline Bias (V) & $\boldsymbol{M}_{\text {diff }}$ & $I_{\text {eq_in }}(\mathrm{A})$ & $I_{\mathrm{obs}}(\mathrm{A})$ & $\langle\boldsymbol{M}\rangle_{\text {dark }}$ & $\left\langle\boldsymbol{M}_{\text {cont_gen }}\right.$ \\
\hline $\mathbf{2 . 5}$ & $\mathbf{5}$ & $5.0 \mathrm{E}-15$ & $4.0 \mathrm{E}-14$ & $\mathbf{3 . 1 3}$ & 2.50 \\
\hline $\mathbf{3 . 5}$ & $\mathbf{1 0}$ & $1.0 \mathrm{E}-14$ & $7.5 \mathrm{E}-13$ & $\mathbf{1 . 3 3}$ & $\mathbf{3 . 9 0}$ \\
\hline $\mathbf{4 . 5}$ & $\mathbf{2 5}$ & $7.0 \mathrm{E}-14$ & $7.0 \mathrm{E}-11$ & $\mathbf{0 . 6 3}$ & 7.45 \\
\hline
\end{tabular}

Table 11. Estimated average dark current generation in a $\mathrm{HgCdTe}$ - $A P D$ with $\lambda_{c}=5.4 \mu \mathrm{m}$ at $\mathrm{T}=80 \mathrm{~K}$

\section{Conclusions}

The latest developments and optimizations of e-APDs and e-APD-FPAs at DEFIR have been reported. The influence of the Cd composition $\left(x_{\mathrm{Cd}}=0.3-0.41\right)$ on the performance in planar homo-junction APDs have been studied at $T=80 \mathrm{~K}$. An exclusive electron multiplication was demonstrated by a high and exponentially increasing gain and low excess noise factor $F=1.2$, even in the e-APDs with the shortest wavelength $\lambda_{\mathrm{c}}=2.9 \mu \mathrm{m}$. Zero-flux FPA measurements allowed to show that the dark current decreases with a decreasing cut-off wavelength, and a record low dark current was observed in the $\lambda_{\mathrm{c}}=2.9 \mu \mathrm{m}$ e-APDs, $I_{\text {eq_in }}=2$ aA at $M=24$. Hence, down to this wavelength, the sensitivity of the e-APDs can be enhanced by increasing the $\mathrm{Cd}$ composition of the multiplication layer, but at a strongly increased reverse bias. The level of dark current obtained in this component is compatible with low flux fast application such as wavefront sensing and fringe tracking in astronomy.

At present, three dedicated e-APD ROICs at a format of 320x256 with a $30 \mu \mathrm{m}$ pitch are developed at DEFIR, in view of high sensitive active and passive imaging. The passive imaging e-APD ROIC is designed for ultra-fast frame rate FPS $>1.5 \mathrm{kHz}$ and low noise $<2$ electrons per frame. This FPA is mainly dedicated to astronomical applications and will be tested during 2010. Two FPA have been designed for active imaging. A first ROIC was designed for dual- 
mode passive and active 2D imaging, with integration times down to $100 \mathrm{~ns}$. This ROIC have been used to characterize the performance of e-APD arrays with wavelengths ranging from $\lambda_{\mathrm{c}}=2.9-5.3 \mu \mathrm{m}$. High performance operability $>99 \%$ was observed at all wavelengths, with values exceeding $99.6 \%$ in cumulated operability of response (average $+/-30 \%$ ) and noise (average $+/-50 \%$ ) at gains $M=10-70$. Theses are the highest value observed so far in e-APD FPAs. In addition, the relative dispersion in gain was systematically lower than $10 \%$.

The implications of the exclusive electrons multiplication in HgCdTe e-APDs for photon counting have been discussed in terms of timing resolution and dark event thresholding. The close to deterministic gain implies that the timing resolution is mainly limited be the collection by the multiplication layer of the electrons generated in the absorption layer. In a recent work, we have shown that if the collection can be accelerated by a built in field, for example due to a compositional gradient in the absorption layer, the timing jitter is below $\Delta t<100 \mathrm{ps}$. The low excess noise factor was shown to enable proportional photon counting and dark current thresholding. Dark current and noise measurements in a $\lambda_{c}=5.4 \mu \mathrm{m}$ e-APD at $T=80 \mathrm{~K}$, have showed that the dark current can be reduced by thresholding at all biases. At high reverse bias, the dominating tunnelling current was found to be close to un-amplified, indicating that high speed and low dark count rate can be achieved at gain in HgCdTe e-APDs, even in the presence of strong tunnelling currents.

\section{Acknowledgments}

The authors which to thank the French MOD (DGA) for its support to the development of the next generation of $\mathrm{HgCdTe}$ FPAs at CEA-LETI/Minatec and Sofradir.

\section{References}

[1] J.D. Beck, C.-F. Wan, M.A. Kinch, J.E. Robinson, Proc. SPIE, 4454, 188 (2001)

[2] J.D. Beck, C.-F. Wan, M.A. Kinch, J.E. Robinson, P. Mitra, R. Scrithfield, F. Ma, J. Campbell, J. Electron. Mater., 35,1166 (2006)

[3] J.D. Beck, C.-F. Wan, M.A. Kinch, J.E. Robinson, P. Mitra, R. Scritchfield, F. Ma, J. Campbell, Proc. SPIE, 5564, 44 (2004)

[4] M.A. Kinch, J.D. Beck, C.-F. Wan, F. Ma, J. Campbell, J. Electron. Mater., 33, 630 (2004)

[5] T. de Lyons, et al., J. Cryst. Growth, 201/202, 980 (1999)

[6] M. Vaidyanathan, et al., 2004 IEEE Aerospace Conference Proc., 1776 (2004)

[7] R. S. Hall, et al., Proc. of SPIE, 5783, 412 (2005)

[8] G. Perrais, J. Rothman, G. Destefanis, J. Baylet, P. Castelein, J.-P. Chamonal et P. Tribolet, SPIE Stockholm 2006.

[9] M. B. Reine, J. W. Marciniec, K. K. Wong, T. Parodos, J. D. Mullarkey, P. A. Lamarre, S. P. Tobin and K. A. Gustavsen, J. Electron. Mater., 36, 1059 (2007)

[10] I. Baker, S. Duncan and J. Copley, Proc. SPIE, 5406, 133 (2004) 
[11] I. Baker, P. Thorne, J. Henderson, J. Copley, D. Humphreys and A. Millar, Proc. SPIE, 6206, 620608-1 (2006)

[12] I. Baker, D. Owton, K. Trundle, P. Thorne, K. Storie, P. Oakley, J. Copley, Proc. SPIE, 6940, 6940$83(2008)$

[13] J. Beck, M. Woodall, R. Scritchfield, M. Ohlson, L. Wood, P. Mitra, and J. Robinson, Proc. SPIE 6542, 654217 (2007)

[14] M. B. Reine, J.W. Marciniec, K.K. Wong, T. Parodos, J. D. Mullarkey, P. A. Lamarre, S. P. Tobin, R.W. Minich and K. A. Gustavsen, J. Electron. Mater., 36, 1059 (2007)

[15] G. Perrais, Jacques Baylet, Gerard Destefanis and Johan Rothman, J. Electron. Mater., 36, 963 (2007)

[16] J. Rothman, G. Perrais, G. Destefanis, J. Baylet, P. Castelein and J.-P. Chamonal, Proc. SPIE, 6542, 654219 (2007)

[17] G. Perrais G. Perrais, J. Rothman, G. Destefanis, J-P. Chamonal, J. Electron. Mater., 37, 1261 (2008)

[18] G. Perrais, S. Derelle, L. Mollard, J.-P. Chamonal, G. Destefanis, G. Vincent, S. Bernhardt and J. Rothman, J. Electron. Mater., 38, 1790 (2009)

[19] J.C. Campbell, et al., IEEE Journ. Of Selec. Top. In Quan. Elec., 10, 777 (2004)

[20] R. B. Emmons, J. Appl. Phys., 38, 3705 (1967)

[21] J. Rothman, G. Perrais, E. de Borniol, P. Castelein, N. Baier, F. Guellec, M. Tchagaspanian, P. Ballet, L. Mollard, S. Gout, A. Perez, M. Fournier, J.-P. Chamonal, P. Tribolet and G. Destefanis, Proc. SPIE 6940, 69402N (2008)

[22] S. Derelle, S. Bernhardt, R. Haidar, J. Deschamps, J. Primot, J. Rothman, S. Rommeluere and N. Guérineau, J. Electron. Mater., 38, 1628 (2009)

[23] J. Rothman, E. de Borniol, P. Ballet, L. Mollard, S. Gout, M. Fournier, J.-P. Chamonal, G. Destefanis, F. Pistone, S. Courtas, X. Lefoule and P. Tribolet, Proc. SPIE 7298, 729834-1(2009)

[24] F. Pistone, P. Tribolet, X. Lefoul, M. Zecri, S. Courtas, P. Jenouvrier and J. Rothman. Proc. SPIE 7298, 729835-1(2009)

[25] F. Guellec, M. Tchagaspanian, E. de Borniol, P. Castelein, A. Perez, J. Rothman, Proc. SPIE 6940, 6940-84 (2008)

[26] J. Rothman, et al, , to be published in J. Electron. Mater., 39, (2010) 